\title{
Estimation over Lossy Networks: A Dynamic Game Approach
}

\author{
Jun Moon and Tamer Başar
}

\begin{abstract}
We study a minimax state estimation $\left(H^{\infty}\right.$ estimation) problem where the dynamical system's disturbance is controlled by an adversary, and measurements from the system to the estimator are lost intermittently according to an i.i.d. Bernoulli process. We first obtain a stochastic minimax state estimator (SMSE) and a stochastic Riccati equation (SRE) that depend on the random measurement arrival process. We then show that the $H^{\infty}$ disturbance attenuation parameter determines the existence of the SMSE. We also analyze the asymptotic behavior of the SRE by showing that the expected value of the SRE is bounded. In particular, we characterize explicit conditions of the disturbance attenuation parameter and the measurement arrival rate above which the expected value of the SRE is bounded. It is also shown that under some conditions, a particular limit of the SMSE is the Kalman filter with intermittent observations but without the disturbance term.
\end{abstract}

\section{INTRODUCTION}

In the past decade, state estimation problems for dynamical systems have been considered under various communication constraints (packet drops, delays, and/or data rate constraints) due to the widespread use of wireless communication and its application to control systems, for instance, networked control systems [1]. Various approaches to state estimation schemes have been proposed in many different settings; see [1] and the references therein. This paper adds to that list by studying a minimax state estimation $\left(H^{\infty}\right.$ estimation) problem where measurements are subject to an i.i.d. Bernoulli-type measurement loss constraint.

Initial study of state estimation under a Bernoulli-type measurement loss model was undertaken in [2] for linear systems. In [2], the stochastic Kalman filter and the associated stochastic error covariance matrix, $P_{k}$, or the Riccati equation generating it, that depend on the random measurement arrival process were obtained. The paper showed that there exists a critical value on the packet arrival rate above which $\mathbb{E}\left\{P_{k}\right\}$, the expected value of the error covariance matrix, becomes bounded. It was also proven that this critical value is a function of the system's unstable eigenvalues and can be expressed in terms of some lower and upper bounds.

The results obtained in [2] have been extended to many different forms [3]-[11]. In [3], a characterization of the critical value was studied when the system eigenvalues are distinct. In [4], it was shown that the lower bound on the critical value determines the boundedness of $\mathbb{E}\left\{P_{k}\right\}$ if the system's observable space is invertible. In [5], a closed-form

Jun Moon is supported by the Fulbright Commission.

This research was supported in part by the U.S. Air Force Office of Scientific Research (AFOSR) MURI grant FA9550-10-1-0573.

All authors are with the Coordinated Science Laboratory, University of Illinois at Urbana-Champaign, Urbana, IL 61801; emails: \{junmoon2, basar1\}@illinois.edu. solution for the critical value was provided for second order systems. In [6], the stability analysis on the error covariance matrix was provided by introducing the notion of the peak covariance process. In [7], the case of two independent partial measurement losses was considered. In [8], [9], the stochastic Riccati equation in [2] was investigated by showing that given any finite $M>0, \mathbb{P}\left\{P_{k} \leq M\right\}$ is bounded. In [10], [11], the authors showed using different methods that the stochastic Riccati equation in [2] converges weakly to a unique stationary distribution.

In this paper, we extend the results of [2] in a substantially different direction by considering an additive unknown disturbance sequence in the state equation and formulating the problem as one of minimax estimation. Hence this can be viewed as an $H^{\infty}$ estimation problem with intermittent observations. The measurement loss is modeled by an i.i.d. Bernoulli process.

In [12], we extended the results in [13] to the $H^{\infty}$ control problem with unknown disturbance controlled by an adversary, under a control packet loss constraint. This paper uses elements of [2] and [12] to obtain some new results for the estimation counterpart of [12]. In particular, we first obtain a stochastic minimax state estimator (SMSE) and a stochastic Riccati equation (SRE) that depend on the random measurement arrival process. We then prove that just as in the case of the deterministic minimax estimation problem, the $H^{\infty}$ disturbance attenuation parameter determines the existence of the SMSE. We also show that depending on the measurement arrival rate and the level of the disturbance attenuation, the expected value of the SRE is bounded; therefore, optimal estimation is guaranteed. In particular, we characterize explicit conditions to guarantee the boundedness of the expected value of the SRE. Finally, we show that the SMSE converges to the Kalman filter in [2] when the disturbance attenuation parameter is infinitely large.

The paper is organized as follows. In Section II, we formulate the problem. In Section III, we obtain the SMSE and the SRE under the Bernoulli-type measurement loss model. Section IV discusses the asymptotic behavior of the expected value of the SRE. Section V provides numerical examples. Finally, in Section VI, we state our conclusions.

\section{PROBLEM Formulation}

Consider the discrete-time linear dynamical system

$$
\begin{aligned}
x_{k+1} & =A x_{k}+D w_{k} \\
y_{k} & =\beta_{k} C x_{k}+E v_{k} \\
z_{k} & =L x_{k},
\end{aligned}
$$


where $x_{k} \in \mathbb{R}^{n}$ is the state; $w_{k} \in \mathbb{R}^{p}$ is the disturbance input; $y_{k} \in \mathbb{R}^{m}$ is the measurement; $v_{k} \in \mathbb{R}^{l}$ is measurement noise; $z_{k} \in \mathbb{R}^{q}$ is a linear combination of $x_{k}$ that has to be estimated; and $A, C, D$, and $L$ are time invariant matrices with appropriate dimensions. In (1), we do not assume any statistics of $w_{k}$ and $v_{k}$ except that they are square summable. The sequence of random variables $\left\{\beta_{k}\right\}$ models the measurement losses between the estimator and the communication channel, and is an i.i.d. Bernoulli process with $\mathbb{P}\left(\beta_{k}=1\right)=\beta$. That is, if $\beta_{k}=1$, then the estimator receives an actual measurement $y_{k}$ from the communication channel, otherwise, there is no new measurement to the estimator. It is also assumed that $(A, D, C)$ are controllable and observable.

We define the information that is available to the estimator at time $k$ as:

$$
\mathcal{I}_{k}:=\left\{\beta_{0: k}, y_{0: k}\right\}:=\left\{\beta_{0}, \ldots, \beta_{k} ; y_{0}, \ldots, y_{k}\right\} .
$$

For system (1), we seek an estimate $\hat{x}_{k}$ (or $\hat{z}_{k}$ ) of the actual state $x_{k}$, to be generated by

$$
\hat{x}_{k}=\pi_{k}\left(\mathcal{I}_{k-1}\right),
$$

where $\pi_{k}$ is an admissible estimator policy that has to be determined. Let $\pi_{0: k}=\left(\pi_{0}, \ldots, \pi_{k}\right)$ be the $k$-tuple of admissible policies and $\Pi$ be the set of the class of admissible policies.

Our main objective in this paper is to find a recursive estimator policy $\pi \in \Pi$ that minimizes the following cost function:

$$
\begin{aligned}
& J(\pi)=\sup _{x_{\bullet}, w_{\bullet}: N-1, v v_{\bullet} N-1} \\
& \frac{\mathbb{E}\left\{\sum_{k=0}^{N-1}\left|z_{k}-\hat{z}_{k}\right|_{Q}^{2}\right\}}{\mathbb{E}\left\{\left|x_{0}-\bar{x}_{0}\right|_{Q \bullet}^{2}+\sum_{k=0}^{N-1}\left|w_{k}\right|^{2}+\beta_{k}\left|v_{k}\right|_{V^{-1}}^{2}\right\}},
\end{aligned}
$$

where $|x|_{S}^{2}:=x^{T} S x$ for some vector $x$ and $S \geq 0 ; Q \geq 0$, $V:=E^{T} E>0, Q_{0}>0$; and $\bar{x}_{0}$ is a known bias term which stands for some initial estimate of $x_{0}$. In (3), if $\beta_{k}=1$ for all $k$, then (3) is the cost function of the deterministic $H^{\infty}$ estimation problem [14], but if $\beta_{k}=0$ for all $k$, then the cost function is identical to that of the open-loop estimation problem. Therefore, the problem can be seen as the $H^{\infty}$ estimation problem with intermittent observations.

Now, with $\gamma>0$, we can introduce the soft-constrained cost function,

$$
\begin{aligned}
& J_{\gamma}\left(\pi, x_{0}, w_{0: N-1}, v_{0: N-1}\right) \\
& =\mathbb{E}\left\{\sum_{k=0}^{N-1}\left|z_{k}-\hat{z}_{k}\right|_{Q}^{2}-\gamma^{2}\left(\left|w_{k}\right|^{2}+\beta_{k}\left|v_{k}\right|_{V^{-1}}^{2}\right.\right. \\
& \left.\left.+\left|x_{0}-\bar{x}_{0}\right|_{Q \bullet}^{2}\right)\right\},
\end{aligned}
$$

where the optimization problem is subject to the system (1).

Since $z_{k}=L x_{k}$, it is natural to let $\hat{z}_{k}=L \hat{x}_{k}$. It is also possible to relax the affine constraint (1b) by substituting $v_{k}=y_{k}-\beta_{k} C x_{k}$ into (4). Then the maximization with respect to $v_{0: k}$ is irrelevant because once $y_{k}$ and $\beta_{k}$ are obtained for each $k$ based on the information structure (3), $v_{k}$ can be uniquely determined by the worse-case optimal values of $x_{0}$ and $w_{k}$. Therefore, the cost function (4) can be reformulated as

$$
\begin{aligned}
& J_{\gamma}\left(\pi, x_{0}, w_{0: N-1}\right) \\
& =\mathbb{E}\left\{\sum_{k=0}^{N-1}\left|x_{k}-\hat{x}_{k}\right|_{\tilde{Q}}^{2}-\gamma^{2}\left(\left|w_{k}\right|^{2}\right.\right. \\
& \left.\left.+\beta_{k}\left|y_{k}-\beta_{k} C x_{k}\right|_{V^{-1}}^{2}\right)-\gamma^{2}\left|x_{0}-\bar{x}_{0}\right|_{Q \bullet}^{2}\right\},
\end{aligned}
$$

where $\tilde{Q}:=L^{T} Q L \geq 0$.

This completes the formulation of the minimax state estimation problem with intermittent observations. Notice that (5) is the cost function of the zero-sum dynamic game and (3) is the disturbance attenuation problem. In what follows, we first obtain an estimator by solving the zero-sum dynamic game (5) and analyze its asymptotic behavior.

\section{Stochastic Minimax State Estimator}

Theorem 1: Consider the zero-sum dynamic game (5) subject to (1a) with $k \in[0, N-1], \beta \in[0,1]$, and a fixed $\gamma>0$.

(i) A stochastic minimax state estimator (SMSE), $\hat{x}_{k}$, exists if

$$
\Sigma_{k}^{-1}-\gamma^{-2} \tilde{Q}>0, \text { almost surely (a.s.), } \forall k,
$$

where $\Sigma_{k}$ is generated by a stochastic Riccati equation (SRE): $\Sigma_{0}=Q_{0}^{-1}$ and

$$
\begin{aligned}
& \Sigma_{k+1} \\
& =A\left(\Sigma_{k}^{-1}-\gamma^{-2} \tilde{Q}+\beta_{k} C^{T} V^{-1} C\right)^{-1} A^{T}+D D^{T} .
\end{aligned}
$$

(ii) The SMSE is

$$
\hat{x}_{k+1}=A \hat{x}_{k}+\beta_{k} A K_{k}\left(y_{k}-C \hat{x}_{k}\right), \hat{x}_{0}=\bar{x}_{0},
$$

where $K_{k}$ is the estimator gain and is expressed as

$$
K_{k}=\left(\Sigma_{k}^{-1}-\gamma^{-2} \tilde{Q}+\beta_{k} C^{T} V^{-1} C\right)^{-1} C^{T} V^{-1}
$$

and $\hat{z}_{k}=L \hat{x}_{k}$.

(iii) The SMSE achieves the disturbance attenuation level $\gamma$, that is $\inf _{\pi \in \Pi} J(\pi)^{1 / 2}=\gamma$.

Proof. We introduce the quadratic cost-to-come (worst past cost) function, $W_{k}\left(x_{k}\right)=\mathbb{E}\left\{-\left|x_{k}-\bar{x}_{k}\right|_{Z_{k}}^{2}+l_{k} \mid \mathcal{I}_{k}\right\}$ where $Z_{k}>0, Z_{0}=\gamma^{2} Q_{0}$, and $l_{0}=0$ [14], [15]. Then the cost from the initial state to stage $k+1$ is

$$
\begin{aligned}
& \mathbb{E}\left\{-\left|x_{k+1}-\bar{x}_{k+1}\right|_{Z_{k+1}}^{2}+l_{k+1} \mid \mathcal{I}_{k+1}\right\} \\
& =\max _{\left(w_{k}, x_{k}\right)} \mathbb{E}\left\{\left\{\left|x_{k}-\hat{x}_{k}\right|_{\tilde{Q}}^{2}-\gamma^{2}\left|w_{k}\right|^{2}\right.\right. \\
& \left.-\gamma^{2} \beta_{k}\left|y_{k}-\beta_{k} C x_{k}\right|_{V^{-1}}^{2}-\left|x_{k}-\bar{x}_{k}\right|_{Z_{k}}^{2}+l_{k} \mid \mathcal{I}_{k+1}\right\},
\end{aligned}
$$

where the maximization is subject to $x_{k+1}=A x_{k}+D w_{k}$. 
Rearranging the preceding equation by using the fact that $\beta_{k}^{3}=\beta_{k}^{2}=\beta_{k}$ gives

$$
\begin{aligned}
& \mathbb{E}\left\{\left|x_{k+1}-\bar{x}_{k+1}\right|_{Z_{k+1}}^{2}-l_{k+1} \mid \mathcal{I}_{k+1}\right\} \\
& =\min _{\left(x_{k}, w_{k}\right)} \mathbb{E}\left\{\left|x_{k}\right|_{U_{k}}^{2}+\gamma^{2}\left|w_{k}\right|^{2}\right. \\
& \left.-2\left(x_{k}^{T} Z_{k} \bar{x}_{k}-x_{k}^{T} \tilde{Q} \hat{x}_{k}+\beta_{k} \gamma^{2} x_{k}^{T} C^{T} V^{-1} y_{k}\right) \mid \mathcal{I}_{k+1}\right\} \\
& +\mathbb{E}\left\{-\left|\hat{x}_{k}\right|_{\tilde{Q}}^{2}+\left|\bar{x}_{k}\right|_{Z_{k}}^{2}+\gamma^{2} \beta_{k}\left|y_{k}\right|_{V-1}^{2}-l_{k} \mid \mathcal{I}_{k+1}\right\},
\end{aligned}
$$

where $U_{k}:=Z_{k}+\beta_{k} \gamma^{2} C^{T} V^{-1} C-\tilde{Q}$.

For the existence of a unique minimizer, by Lemma 6.1 in [14], given $\mathcal{I}_{k}$, it is necessary to have $Z_{k}-\tilde{Q}>0$ for all $k$. Then by Lemma 6.2 in [14], the cost at stage $k+1$ is

$$
\begin{aligned}
& \mathbb{E}\left\{\left|x_{k+1}-\bar{x}_{k+1}\right|_{Z_{k+1}}^{2}-l_{k+1} \mid \mathcal{I}_{k+1}\right\} \\
& =\mathbb{E}\left\{\mid x_{k+1}-A U_{k}^{-1}\left(Z_{k} \bar{x}_{k}+\beta_{k} \gamma^{2} C^{T} V^{-1} y_{k}\right.\right. \\
& \left.-\tilde{Q} \hat{x}_{k}\right)\left.\right|_{M_{k}^{-1}} ^{2}+\left|\tilde{Q} \hat{x}_{k}-Z_{k} \bar{x}_{k}-\beta_{k} \gamma^{2} C^{T} V^{-1} y_{k}\right|_{U_{k}^{-1}}^{2} \\
& \left.+\left|\bar{x}_{k}\right|_{Z_{k}}^{2}-\left|\hat{x}_{k}\right|_{\tilde{Q}}^{2}+\gamma^{2} \beta_{k}\left|y_{k}\right|_{V-1}^{2}-l_{k} \mid \mathcal{I}_{k+1}\right\},
\end{aligned}
$$

where $M_{k}:=A U_{k}^{-1} A^{T}+\gamma^{-2} D D^{T}$.

Since this is true for all $k$, the dynamic equation for $\bar{x}_{k}$ can be written as

$$
\begin{aligned}
& \bar{x}_{k+1}=A U_{k}^{-1}\left(Z_{k} \bar{x}_{k}+\beta_{k} \gamma^{2} C^{T} V^{-1} y_{k}-\tilde{Q} \hat{x}_{k}\right) \\
& Z_{k+1}=\left(A U_{k}^{-1} A^{T}+\gamma^{-2} D D^{T}\right)^{-1} .
\end{aligned}
$$

Also with $Z_{0}=\gamma^{2} Q_{0}$, let $\Sigma_{k}:=\gamma^{2} Z_{k}^{-1}$. Then (7) can be rewritten as

$$
\bar{x}_{k+1}=A \bar{x}_{k}+\beta_{k} A K_{k}\left[\left(y_{k}-C \bar{x}_{k}\right)+\left(\gamma^{-2} \tilde{Q}\left(\bar{x}_{k}-\hat{x}_{k}\right)\right)\right] .
$$

Now, choose the estimator policy to be the certainty equivalence policy $\hat{x}_{k}=\bar{x}_{k}$ for all $k$, which can be obtained by

$$
\hat{x}_{k}=\arg \max _{x_{k}} \mathbb{E}\left\{-\gamma^{2}\left|x_{k}-\bar{x}_{k}\right|_{\Sigma_{k}^{-1}}^{2}+l_{k} \mid \mathcal{I}_{k}\right\} .
$$

Then we have the SMSE and the SRE.

To prove part (iii), note that under (6), by the definition of the cost-to-come function [15], $W_{k}\left(x_{k}\right) \leq 0$ for all disturbance $\left(x_{0}, w_{0: N-1}\right)$ and for all $k$. This implies that the value of the zero-sum game (5) is finite and is bounded from above by 0 . Hence, the SMSE achieves the disturbance attenuation level $\gamma$. Observe that if (6) does not hold at $\bar{k} \in[0, N-1]$, the zero-sum game is unbounded since $W_{k}\left(x_{k}\right)$ can be made arbitrarily large by an appropriate choice of the disturbance, which shows that $\gamma$ cannot be the disturbance attenuation level for the SMSE. This completes the proof of the theorem.

Remark 1: The evolution of the SMSE and the SRE depend on $\beta_{k}$; therefore, they are random variables being a function of $\beta_{k}$, which itself is random. Moreover, as $\gamma \rightarrow \infty$, (6) holds and the SRE converges to $P_{k}$ in [2].

Note that the SMSE uses a sequence of the measurement arrival information, $\left\{\beta_{k}\right\}$, and a sequence of measurements, $\left\{y_{k}\right\}$, to perform the worst-case estimation; therefore it is time-varying and stochastic. On the other hand, the $H^{\infty}$ Markov jump linear estimator (MJLE) in [16] uses only the last arrival $\beta_{k}$ and $\left\{y_{k}\right\}$. This implies that the $H^{\infty}$ MJLE is stochastic but time-invariant and therefore is suboptimal. It is easy to expect that the SMSE provides a better estimation performance than that of the $H^{\infty}$ MJLE, since it exploits additional information regarding the measurement arrival.

For the infinite-horizon case $(N \rightarrow \infty$ of (5)), if (6) holds for all $k$, then the SMSE still can achieve the disturbance attenuation level $\gamma$. To be specific, for a fixed $\beta$, define

$$
\gamma^{*}(\beta):=\inf \{\gamma>0: \text { (6) holds a.s. } \forall k\} \text {. }
$$

If $\gamma^{*}(\beta)$ is finite, then for any $\gamma>\gamma^{*}(\beta)$, the disturbance attenuation level for the SMSE is $\gamma$. Note that $\gamma^{*}(0)$ is not finite when $A$ is unstable since this is equivalent to the openloop estimation problem of the unstable system.

\section{ASYMPTOTIC ANALYSis ON $\mathbb{E}\left\{\Sigma_{k}\right\}$}

In this section, we provide a complete analysis of the asymptotic behavior of the stochastic Riccati equation (SRE) obtained in Theorem 1. We will show that under some conditions, $\mathbb{E}\left\{\Sigma_{k}\right\}$ is bounded for all $k$. In the rest of this paper, we take $\tilde{Q}=Q$.

\section{A. Modified Lyapunov and Riccati Equations}

Consider the modified Lyapunov and Riccati equations

$$
\begin{aligned}
\breve{\Sigma}_{k+1} & =(1-\beta) A \breve{\Sigma}_{k} A^{T}+D D^{T} \\
\bar{\Sigma}_{k+1} & =(1-\beta)\left(A\left(\bar{\Sigma}_{k}^{-1}-\gamma^{-2} Q\right)^{-1} A^{T}+D D^{T}\right) \\
& +\beta\left(A\left(\bar{\Sigma}_{k}^{-1}-\gamma^{-2} Q+C^{T} V^{-1} C\right)^{-1} A^{T}+D D^{T}\right),
\end{aligned}
$$

where $\breve{\Sigma}_{0}=\bar{\Sigma}_{0}=Q_{0}^{-1}$. Later we will prove that (8a) and (8b) generate lower and upper bounds on $\mathbb{E}\left\{\Sigma_{k}\right\}$ if $\gamma$ and $\beta$ are in certain ranges. Before stating our main results, we first discuss convergence properties of (8a) and (8b).

Theorem 2: (8a) is a non-decreasing sequence. Suppose $\breve{\Sigma}^{+}:=\breve{\Sigma}^{+}(\beta)>0$ solves (8a). Then $\lim _{k \rightarrow \infty} \breve{\Sigma}_{k}=\breve{\Sigma}^{+}$. The existence condition of $\breve{\Sigma}^{+}$is $\rho(\tilde{A}):=\max _{i}\left|\lambda_{i}(\tilde{A})\right|<1$ where $\lambda(\tilde{A})$ is eigenvalue of $\tilde{A}=\sqrt{1-\beta} A$. Finally, letting $\breve{\beta}_{c}:=1-1 / \rho^{2}(A)$, if $\beta>\breve{\beta}_{c}$, then $\breve{\Sigma}^{+}(\beta)$ solves (8a).

Theorem 3: Let $\gamma>0$ be fixed. Under the condition

$$
\bar{\Sigma}_{k}-\gamma^{-2} Q>0,
$$

(8b) is a monotonically non-decreasing sequence. Also if there is a positive definite matrix $\bar{\Sigma}:=\bar{\Sigma}(\beta, \gamma)$ such that $\bar{\Sigma} \geq \bar{\Sigma}_{k}$ for all $k \geq 0$, then (8b) has a limit, $\bar{\Sigma}^{+}:=\bar{\Sigma}^{+}(\beta, \gamma)$, that is positive definite and a fixed point of the following algebraic Riccati equation (ARE)

$$
\begin{aligned}
\bar{\Sigma} & =(1-\beta)\left(A\left(\bar{\Sigma}^{-1}-\gamma^{-2} Q\right)^{-1} A^{T}+D D^{T}\right) \\
& +\beta\left(A\left(\bar{\Sigma}^{-1}-\gamma^{-2} Q+C^{T} V^{-1} C\right)^{-1} A^{T}+D D^{T}\right),
\end{aligned}
$$

satisfying

$$
\bar{\Sigma}^{-1}-\gamma^{-2} Q>0
$$

Conversely, if there is a positive definite matrix, $\bar{\Sigma}^{+}$, that solves (10) and satisfies (11), then (8b) converges to $\bar{\Sigma}^{+}$. 
Proof. It is easy to see that (8b) is a monotonically nondecreasing positive definite matrix sequence with condition (9). Thus if $\bar{\Sigma} \geq \bar{\Sigma}_{k}$ for all $k$, then (8b) is a convergent matrix sequence and under the controllability and observability conditions, the limit $\bar{\Sigma}^{+}$is positive definite. Then, by continuity, $\bar{\Sigma}^{+}$is a solution of (10) that satisfies (11). The converse statement follows from the fact that (8b) is a monotonically non-decreasing positive definite matrix sequence with condition (9) and continuity of the Riccati equation (8b).

In order to characterize conditions that guarantee boundedness of $\mathbb{E}\left\{\Sigma_{k}\right\}$, we need to know the smallest value of $\beta$ and $\gamma, \bar{\beta}_{c}(\gamma)$ and $\bar{\gamma}^{*}(\beta)$, above which (8a) converges; hence (10) has a solution that satisfies (11). By adopting the argument in [12], we can define those parameters as follows:

Proposition 1: Suppose $\beta$ is fixed. Let $\bar{\Gamma}(\beta):=\{\gamma>$ $\left.0:\left(\bar{\Sigma}(\beta, \gamma)-\gamma^{-2} Q\right)>0\right\}$. Then $\bar{\gamma}^{*}(\beta):=\inf \{\gamma: \gamma \in$ $\bar{\Gamma}(\beta)\}$. Moreover, if the set $\bar{\Gamma}(\beta)$ is not empty with $\beta_{1}$ and $\beta_{2}$, and we have $\beta_{1}>\beta_{2}$, then $\bar{\gamma}^{*}\left(\beta_{1}\right)<\bar{\gamma}^{*}\left(\beta_{2}\right)$.

Proof. Observe that $\bar{\Gamma}(0)$ is empty when $A$ is unstable. Since (9) guarantees monotonicity of $(8 b), \bar{\gamma}^{*}(\beta)$ follows. The second statement follows from Lemma 1(i).

Proposition 2: Suppose $\gamma>\bar{\gamma}^{*}(1)$ is fixed. Let $\bar{\Lambda}(\gamma):=$ $\left\{0 \leq \beta \leq 1:\left(\bar{\Sigma}(\beta, \gamma)-\gamma^{-2} Q\right)>0\right\}$. Then $\bar{\beta}_{c}(\gamma):=$ $\inf \{\beta: \beta \in \bar{\Lambda}(\gamma)\}$. Moreover, if $\gamma_{1}>\gamma_{2}$, then $\bar{\beta}_{c}\left(\gamma_{1}\right)<$ $\bar{\beta}_{c}\left(\gamma_{2}\right)$. Also as $\gamma \rightarrow \infty, \bar{\beta}_{c}(\gamma)$ converges to $\bar{\lambda}$ where $\bar{\lambda}$ is defined in [2] that ensures the boundedness of $\mathbb{E}\left\{P_{k}\right\}$.

Proof. With a fixed $\gamma$, the only case when (10) does not admit a solution is when (10) does not have any solutions that satisfy (11). Therefore, $\bar{\beta}_{c}(\gamma)$ is true. The second and last statements follow from Lemma 1(ii) and (vi), respectively. $\square$

Remark 2: From Theorem 1 and previous results, if $\gamma>$ $\bar{\gamma}^{*}(\beta)$ and $\beta>\bar{\beta}_{c}(\gamma)$, then $\left\{\bar{\Sigma}_{k}\right\} \rightarrow \bar{\Sigma}^{+}$as $k \rightarrow \infty$.

\section{B. Boundedness of $\mathbb{E}\left\{\Sigma_{k}\right\}$}

The next theorem shows the existence of a critical value that determines the boundedness of $\mathbb{E}\left\{\Sigma_{k}\right\}$.

Theorem 4: Suppose $\gamma^{*}(\beta)$ is finite. For all $\gamma>\gamma^{*}(\beta)$, there exists a critical value $\beta_{c}$ such that with $\mathbb{E}\left\{\Sigma_{0}\right\}=Q_{0}^{-1}$, $\lim _{k \rightarrow \infty} \mathbb{E}\left\{\Sigma_{k}\right\}=\infty$ if $\beta \leq \beta_{c}$ and $\mathbb{E}\left\{\Sigma_{k}\right\} \leq M_{\Sigma_{\bullet}}(\gamma)$ for all $k \geq 0$ if $\beta>\beta_{c}$ where $M_{\Sigma_{\bullet}}(\gamma)$ depends on the initial condition $\Sigma_{0}$ and $\gamma$.

Proof. Suppose $A$ is stable; then $\mathbb{E}\left\{\Sigma_{k}\right\}$ is bounded for all $\beta \in[0,1]$ since the SRE reduces to the deterministic $H^{\infty}$ Riccati equation when $\beta=1$ and is the modified Lyapunov equation under (6) when $\beta=0$. Therefore, we consider the case when $A$ is unstable. If $\beta=1$, then under the controllability and observability assumptions, $\mathbb{E}\left\{\Sigma_{k}\right\}$ converges to a positive definite matrix. If $\beta=0$, then $\mathbb{E}\left\{\Sigma_{k}\right\} \rightarrow \infty$ as $k \rightarrow \infty$. Now, suppose that there is $\beta_{1}$ such that $\mathbb{E}\left\{\Sigma_{k}\right\}$ is bounded. Clearly, by Lemma $1(\mathrm{i}), \mathbb{E}\left\{\Sigma_{k}\right\}$ is bounded for all $\beta>\beta_{1}$. In fact, if $\beta_{2}>\beta_{1}$, then we have

$$
\begin{aligned}
& \mathbb{E}_{\beta_{1}}\left\{\Sigma_{k+1}\right\} \\
&=\mathbb{E}_{\beta_{1}}\left\{A\left(\Sigma_{k}^{-1}-\gamma^{-2} Q+\beta_{k} C^{T} V^{-1} C\right)^{-1} A^{T}+D D^{T}\right\} \\
&=\left(1-\beta_{1}\right) \mathbb{E}\left\{A\left(\Sigma_{k}^{-1}-\gamma^{-2} Q\right)^{-1} A^{T}+D D^{T}\right\} \\
&+\beta_{1} \mathbb{E}\left\{A\left(\Sigma_{k}^{-1}-\gamma^{-2} Q+C^{T} V^{-1} C\right)^{-1} A^{T}+D D^{T}\right\} \\
& \geq\left(1-\beta_{2}\right) \mathbb{E}\left\{A\left(\Sigma_{k}^{-1}-\gamma^{-2} Q\right)^{-1} A^{T}+D D^{T}\right\} \\
&+\beta_{2} \mathbb{E}\left\{A\left(\Sigma_{k}^{-1}-\gamma^{-2} Q+C^{T} V^{-1} C\right)^{-1} A^{T}+D D^{T}\right\} \\
&= \mathbb{E}_{\beta_{2}}\left\{\Sigma_{k+1}\right\},
\end{aligned}
$$

where Lemma 1(i) is used to obtain the inequality. Finally, define $\beta_{c}:=\inf \left\{\beta \in[0,1]: \mathbb{E}_{\beta}\left\{\Sigma_{k}\right\}\right.$ is bounded. $\}$. Note that the bound is a function of $\gamma$ since the SRE is parameterized by $\gamma$. This completes the proof.

Just as in the case of the Kalman filter discussed in [2], the exact value of $\beta_{c}$ is hard to compute but it is possible to obtain lower and upper bounds.

Theorem 5: Suppose $\gamma^{*}(\beta)$ and $\bar{\gamma}^{*}(\beta)$ are finite. If $\gamma>$ $\gamma^{*}(\beta)$ and $\gamma>\bar{\gamma}^{*}(\beta)$, then the critical value $\beta_{c}$ satisfies $\breve{\beta}_{c} \leq \beta_{c} \leq \bar{\beta}_{c}(\gamma)$.

Proof. We first show that $\breve{\Sigma}_{k} \leq \mathbb{E}\left\{\Sigma_{k}\right\} \leq \bar{\Sigma}_{k}$ for all $k$. To prove the first inequality, we use induction. $\breve{\Sigma}_{k} \leq \mathbb{E}\left\{\Sigma_{k}\right\}$ implies

$$
\begin{aligned}
& \breve{\Sigma}_{k+1} \\
& =(1-\beta) A \breve{\Sigma}_{k} A^{T}+D D^{T} \\
& \stackrel{(\text { a) }}{\leq}(1-\beta) A \mathbb{E}\left\{\Sigma_{k}\right\} A^{T}+D D^{T} \\
& \stackrel{\text { (b) }}{=} \mathbb{E}\left\{(1-\beta) A \Sigma_{k} A^{T}+D D^{T}\right\} \\
& \stackrel{\text { (c) }}{\leq}(1-\beta) \mathbb{E}\left\{A\left(\Sigma_{k}^{-1}-\gamma^{-2} Q\right)^{-1} A^{T}+D D^{T}\right\} \\
& \quad+\beta \mathbb{E}\left\{A\left(\Sigma_{k}^{-1}-\gamma^{-2} Q+C^{T} V^{-1} C\right)^{-1} A^{T}+D D^{T}\right\} \\
& \stackrel{(\text { d) }}{=} \mathbb{E}\left\{A\left(\Sigma_{k}^{-1}-\gamma^{-2} Q+\beta_{k} C^{T} V^{-1} C\right)^{-1} A^{T}+D D^{T}\right\} \\
& =\mathbb{E}\left\{\Sigma_{k+1}\right\},
\end{aligned}
$$

where (a) follows from the induction argument, (b) is the linearity of the expectation, (c) is Lemma $1(\mathrm{v})$, and (d) is due to law of iterated expectations.

For the second part, $\mathbb{E}\left\{\Sigma_{k}\right\} \leq \bar{\Sigma}_{k}$ implies

$$
\begin{aligned}
& \mathbb{E}\left\{\Sigma_{k+1}\right\} \\
& =\mathbb{E}\left\{A\left(\Sigma_{k}^{-1}-\gamma^{-2} Q+\beta_{k} C^{T} V^{-1} C\right)^{-1} A^{T}+D D^{T}\right\} \\
& \stackrel{(\mathrm{d})}{=}(1-\beta) \mathbb{E}\left\{A\left(\Sigma_{k}^{-1}-\gamma^{-2} Q\right)^{-1} A^{T}+D D^{T}\right\} \\
& \quad+\beta \mathbb{E}\left\{A\left(\Sigma_{k}^{-1}-\gamma^{-2} Q+C^{T} V^{-1} C\right)^{-1} A^{T}+D D^{T}\right\} \\
& \stackrel{(\mathrm{e})}{\leq} \\
& \quad(1-\beta)\left(A\left(\mathbb{E}\left\{\Sigma_{k}\right\}^{-1}-\gamma^{-2} Q\right)^{-1} A^{T}+D D^{T}\right) \\
& \quad+\beta\left(A\left(\mathbb{E}\left\{\Sigma_{k}\right\}^{-1}-\gamma^{-2} Q+C^{T} V^{-1} C\right)^{-1} A^{T}+D D^{T}\right) \\
& \stackrel{\text { (f) }}{\leq}(1-\beta)\left(A\left(\bar{\Sigma}_{k}^{-1}-\gamma^{-2} Q\right)^{-1} A^{T}+D D^{T}\right) \\
& \quad+\beta\left(A\left(\bar{\Sigma}_{k}^{-1}-\gamma^{-2} Q+C^{T} V^{-1} C\right)^{-1} A^{T}+D D^{T}\right) \\
& =\bar{\Sigma}_{k+1},
\end{aligned}
$$

where (d) is due to the law of iterated expectations, (e) follows by Jensen's inequality since by Lemma 1(iv), the 
equation inside the bracket is a concave function in $\Sigma_{k}$, and (f) is obtained from the induction argument.

Now, if $\beta<\breve{\beta}_{c}$, then by Theorem $2,\left\{\breve{\Sigma}_{k}\right\} \rightarrow \infty$ as $k \rightarrow \infty$ and therefore, $\mathbb{E}\left\{\Sigma_{k}\right\} \rightarrow \infty$ for all initial conditions, which shows $\beta_{c} \geq \breve{\beta}_{c}$. Let us consider the case $\beta>\bar{\beta}_{c}(\gamma)$. By Theorem 3 and the previous inequality, we have $\left\{\bar{\Sigma}_{k}\right\} \rightarrow$ $\bar{\Sigma}$ as $k \rightarrow \infty$ and $\bar{\Sigma} \geq \mathbb{E}\left\{\Sigma_{k}\right\}$ for all $k$ and the initial condition. This implies $\beta_{c} \leq \bar{\beta}_{c}(\gamma)$; thus completing the proof.

Theorem 5 provides us with a range of values for $\beta$ that guarantees the boundedness of $\mathbb{E}\left\{\Sigma_{k}\right\}$. The next theorem provides lower and upper bounds on $\mathbb{E}\left\{\Sigma_{k}\right\}$.

Theorem 6: Assume that $\gamma>\gamma^{*}(\beta)$ and $\gamma>\bar{\gamma}^{*}(\beta)$ are finite. If $\beta>\bar{\beta}_{c}(\gamma)$, then $\breve{\Sigma}_{k} \leq \mathbb{E}\left\{\Sigma_{k}\right\} \leq \bar{\Sigma}_{k}$ for all $k$. Moreover, $\lim _{k \rightarrow \infty} \breve{\Sigma}_{k}=\breve{\Sigma}^{+}$and $\lim _{k \rightarrow \infty} \overline{\bar{\Sigma}}_{k}=\bar{\Sigma}^{+}$.

Proof. This result follows from Theorem 5 and the preceding discussion.

Remark 3: In Theorem 6, (8a) is exactly identical to the lower bound on $\mathbb{E}\left\{P_{k}\right\}$ in [2]. As for the upper bound on $\mathbb{E}\left\{\Sigma_{k}\right\}$, by Lemma 1 (vi), as $\gamma \rightarrow \infty$, it converges to the upper bound on $\mathbb{E}\left\{P_{k}\right\}$. Furthermore, just as in the case of the deterministic $H^{\infty}$ estimation problem, the SMSE converges to the Kalman filter in [2] as $\gamma \rightarrow \infty$.

Remark 4: It was shown in [4] that for the Kalman filtering problem in [2], if the system has an invertible observable subspace or $C$ is invertible, the lower critical value determines the boundedness of $\mathbb{E}\left\{P_{k}\right\}$. However, this is not true for the SMSE unless $\gamma$ is sufficiently large. This is because the SMSE also considers disturbance attenuation that causes increasing $\bar{\beta}(\gamma)$ due to Proposition 2 and Theorem 6. This fact will be demonstrated in Section V.

\section{NUMERICAL EXAMPLES}

The first simulation is conducted to compare bounds on $\mathbb{E}\left\{\Sigma_{k}\right\}$ with that of $\mathbb{E}\left\{P_{k}\right\}$ where $P_{k}$ is the error covariance matrix in [2]. To allow for a comparison, we use the same linear system model as in [2].

Consider a scalar system where $A=-1.25, C=1, D=$ $1, V=E^{2}=2.5$, and $Q=1$. Note that since $C$ is scalar, for the Kalman filter in [2], the lower critical value, 0.36 , decides the boundedness of $\mathbb{E}\left\{P_{k}\right\}$. To see the boundedness of $\mathbb{E}\left\{\Sigma_{k}\right\}$, by Theorem 6 , we need $\gamma>\bar{\gamma}^{*}(\beta)$, and $\beta>$ $\bar{\beta}_{c}(\gamma)$. Figure 1(top) is a plot of the convergence region of (8b). Observe that $\bar{\beta}_{c}(\gamma) \rightarrow 0.36$ as $\gamma \rightarrow \infty$, and $\bar{\gamma}^{*}(1)=$ 1.585. Figure 1(bottom) shows a plot of the steady state of the upper and lower bounds on $\mathbb{E}\left\{\Sigma_{k}\right\}$ versus $\beta$. It shows that although $C$ is invertible, since $\bar{\beta}_{c}(\gamma)=0.36$ only when $\gamma$ is sufficiently large, $\breve{\beta}_{c}$ is not the actual critical value for optimal estimation. This result is due to the fact that the SMSE also considers the disturbance attenuation that results in increasing the critical value. In other words, the SMSE needs the more reliable measurement connection for the high disturbance attenuation performance. Note that if $\gamma>\gamma^{*}(\beta)$, we obtain the boundedness of $\mathbb{E}\left\{\Sigma_{k}\right\}$.
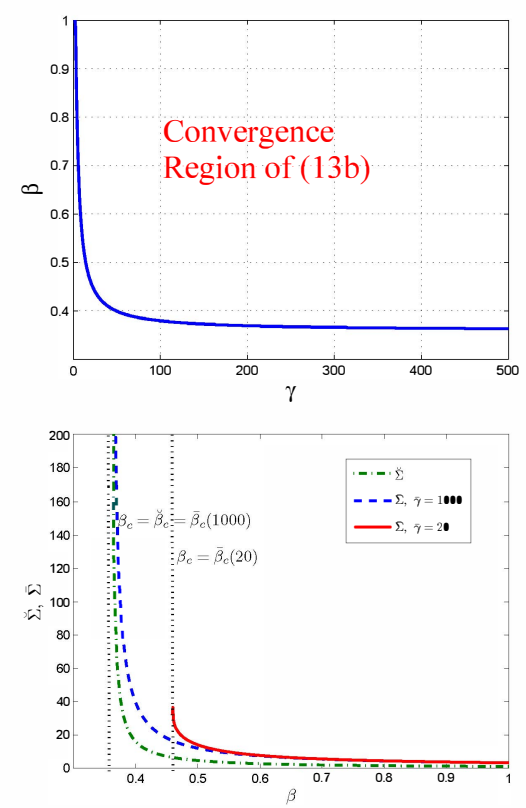

Fig. 1. Convergence region of the Riccati equation (8b) (top) and transition to instability (bottom) for the scalar system.

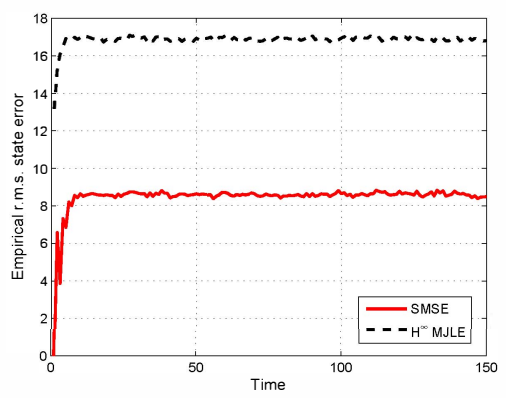

Fig. 2. Empirical r.m.s. estimation errors of the scalar system with $w_{k}=$ 10 and $v_{k}=-10$.

Figure 2 shows the root mean square (r.m.s.) estimation errors of the scalar system for the SMSE and the $H^{\infty}$ MJLE in [16] with $w_{k}=10$ and $v_{k}=-10$. The $H^{\infty}$ MJLE is obtained by MATLAB YALMIP where the minimum disturbance attenuation performance level is 7.5. We use $\gamma=50$ for the SMSE. Each estimation error is obtained by averaging 10000 Monte Carlo simulations. As can be seen, the SMSE outperforms the $H^{\infty}$ MJLE because while the SMSE is an optimal time-varying estimator for every measurement case due to the information structure (2), the $H^{\infty}$ MJLE is suboptimal since it uses the static estimator gain depending on $\beta_{k}$. Note that although $\gamma$ for the SMSE is larger than that of the $H^{\infty}$ MJLE, the reduced estimation error is obtained by the SMSE. Note finally that as $\gamma \rightarrow \infty$, the performance of the SMSE will be identical to that of the Kalman filter case in [2].

\section{COnClusions And Future Work}

In this paper, we have presented the stochastic minimax state estimator (SMSE) under the Bernoulli-type measure- 
ment loss model. The problem formulation can be viewed as $H^{\infty}$ estimation with intermittent observations. If the measurement loss rate and the disturbance attenuation parameter satisfy certain threshold conditions, optimal estimation is guaranteed in the sense that the expected value of the stochastic Riccati equation (SRE) is bounded. We have also provided exact lower and upper bounds on the expected value of the SRE. Finally, it has been proven that just as in the case of the deterministic minimax estimation problem, as $\gamma \rightarrow \infty$, the SMSE converges to the Kalman filter in [2]. An important outcome of the study of this paper is that the SMSE requires more reliable measurement connection if we focus on the disturbance attenuation performance.

Future work will involve a rigorous analysis of the SRE. In particular, it is possible to use different performance metrics as in [8]-[11] to arrive at boundedness of $\mathbb{P}\left(\Sigma_{k} \leq M\right)$ and weak convergence of the SRE. Moreover, it is possible to generalize the packet drop model by using the Gilbert-Elliott model in [6].

\section{APPENDIX}

We rewrite the algebraic Riccati equation (ARE) (10):

$$
\begin{aligned}
X & =(1-\beta)\left(A\left(X^{-1}-\eta^{-2} Q\right)^{-1} A^{T}+D D^{T}\right) \\
& +\beta\left(A\left(X^{-1}-\eta^{-2} Q+C^{T} V^{-1} C\right)^{-1} A^{T}+D D^{T}\right) .
\end{aligned}
$$

The following lemma provides useful properties of (A.12).

Lemma 1: Suppose $(A, C)$ is observable, $(A, D)$ is controllable, $X>0, Q=G^{T} G \geq 0$, and $\eta>0$. Suppose $X$ and $\eta$ satisfy $\left(X^{-1}-\eta^{-2} Q\right)>0$.

(i) (A.12) is decreasing in $\beta$.

(ii) (A.12) is decreasing in $\eta$.

(iii) Suppose $\rho \in[0,1]$ and $X:=\rho X_{1}+(1-\rho) X_{2}$ where $X_{1}, X_{2}>0$. Then

$$
\begin{aligned}
& A\left(X^{-1}-\eta^{-2} Q\right)^{-1} A^{T}+D D^{T} \\
& A\left(X^{-1}-\eta^{-2} Q+C^{T} V^{-1} C\right)^{-1} A^{T}+D D^{T}
\end{aligned}
$$

are concave in $X$.

(iv) The ARE (A.12) is concave in $X$.

(v) We have the following inequalities:

$$
\begin{aligned}
& (1-\beta) A X A^{T}+D D^{T} \\
& \leq(1-\beta)\left(A\left(X^{-1}-\eta^{-2} Q\right)^{-1} A^{T}+D D^{T}\right) \\
& \quad+\beta\left(A\left(X^{-1}-\eta^{-2} Q+C^{T} V^{-1} C\right)^{-1} A^{T}+D D^{T}\right) .
\end{aligned}
$$

(vi) As $\gamma \rightarrow \infty$, (A.12) converges to

$$
X=A X A^{T}+D D^{T}-\beta A X C^{T}\left(C X C^{T}+V\right)^{-1} C X A^{T} \text {. }
$$

Proof. (i) For $\beta_{2}>\beta_{1}$, consider

$$
\begin{aligned}
& \left(\beta_{2}-\beta_{1}\right)(\underbrace{A\left(X^{-1}-\eta^{-2} Q\right)^{-1} A^{T}+D D^{T}}_{\text {(a) }} \\
& -\underbrace{A\left(X^{-1}-\eta^{-2} Q+C^{T} V^{-1} C\right)^{-1} A^{T}+D D^{T}}_{\text {(b) }}) .
\end{aligned}
$$

Since $V>0$ and $C \neq 0$, under $\left(X^{-1}-\eta^{-2} Q\right)>0$, (a) $>$ (b); thus, completing the proof. (ii) The result is immediate.

(iii) By applying the matrix inversion lemma, (A.13) can be written as

$$
A X A^{T}+A X G^{T}\left(\eta^{2} I-G X G^{T}\right)^{-1} G X A^{T}+D D^{T} .
$$

The preceding equation is dual of the problem of maximizing

$$
\sum_{k=0}^{\infty} x_{k}^{T} G^{T} G x_{k}-\eta^{2} w_{k}^{T} w_{k}
$$

subject to (1a). Since $\left(X^{-1}-\eta^{-2} Q\right)>0$ implies $\left(\eta^{2} I-G X G^{T}\right)>0$, the first result follows. The second result follows from the fact that it is dual of the deterministic $H^{\infty}$ Riccati equation [14].

(iv) It follows from part (iii) and the fact that (A.12) is the sum of two concave functions.

(v) The result is immediate.

(vi) It can be shown by using the matrix inversion lemma. $\square$

\section{REFERENCES}

[1] J. Hespanha, P. Naghshtabrizi, and Y. Xu, "A survey of recent results in networked control systems," Proc. of the IEEE, vol. 95, no. 1, pp. 138-162, Jan. 2007.

[2] B. Sinopoli, L. Schenato, M. Franceschetti, K. Poolla, M. Jordan, and S. Sastry, "Kalman filtering with intermittent observations," IEEE Trans. on Autom. Control, vol. 49, no. 9, pp. 1453-1464, Sept. 2004.

[3] Y. Mo and B. Sinopoli, "A characterization of the critical value for Kalman filtering with intermittent observations," in Proc. 47th Conf. on Decision and Control (CDC), Dec. 2008, pp. 2692-2697.

[4] K. Plarre and F. Bullo, "On Kalman filtering for detectable systems with intermittent observations," IEEE Trans. on Autom. Control, vol. 54, no. 2, pp. 386-390, Feb. 2009.

[5] Y. Mo and B. Sinopoli, "Kalman filtering with intermittent observations: Critical value for second order system," in Proc. of 18th IFAC World Congress, 2011.

[6] M. Huang and S. Dey, "Stability of Kalman filtering with Markovian packet losses," Automatica, vol. 43, no. 4, pp. 598 - 607, 2007.

[7] X. Liu and A. Goldsmith, "Kalman filtering with partial observation losses," in Proc. 43rd Conf. on Decision and Control (CDC), Dec. 2004, pp. 4180-4186.

[8] L. Shi, M. Epstein, and R. Murray, "Kalman filtering over a packetdropping network: A probabilistic perspective," IEEE Trans. on Autom. Control, vol. 55, no. 3, pp. 594-604, March 2010.

[9] M. Epstein, L. Shi, A. Tiwari, and R. M. Murray, "Probabilistic performance of state estimation across a lossy network," Automatica, vol. 44, no. 12, pp. 3046 - 3053, 2008.

[10] A. Censi, "Kalman filtering with intermittent observations: Convergence for semi-markov chains and an intrinsic performance measure," IEEE Trans. on Autom. Control, vol. 56, no. 2, pp. 376-381, 2011.

[11] S. Kar, B. Sinopoli, and J. M. F. Moura, "Kalman filtering with intermittent observations: Weak convergence to a stationary distribution," IEEE Trans. on Autom. Control, vol. 57, no. 2, pp. 405 - 420, Feb. 2012.

[12] J. Moon and T. Başar, "Control over TCP-like lossy networks: A dynamic game approach," in Proc. of American Control Conference, Washington, DC, June 2013, pp. 1581-1586.

[13] O. C. Imer, S. Yüksel, and T. Başar, "Optimal control of LTI systems over unreliable communication links," Automatica, vol. 42, no. 9, pp. 1429-1439, 2006.

[14] T. Başar and P. Bernhard, $H^{\infty}$ Optimal Control and Related Minimax Design Problems, 2nd ed. Boston, MA: Birkhäuser, 1995.

[15] G. Didinsky, "Design of minimax controllers for nonlinear systems using cost-to-come methods," Ph.D. dissertation, University of Illinois, Urbana-Champaign, IL, 1994.

[16] A. Goncalves, A. Fioravanti, and J. Geromel, " $\mathrm{H}^{\infty}$ filtering of discrete-time Markov jump linear systems through linear matrix inequalities," IEEE Trans. on Autom. Control, vol. 54, no. 6, pp. 13471351, 2009. 